\title{
ON THE LOSS OF COMPACTNESS IN THE VECTORIAL HETEROCLINIC CONNECTION PROBLEM
}

\author{
NIKOS KATZOURAKIS
}

\begin{abstract}
We give an alternative proof of the theorem of Alikakos-Fusco [AF] concerning existence of heteroclinic solutions $U: \mathbb{R} \longrightarrow \mathbb{R}^{N}$ to the system

$$
\left\{\begin{array}{l}
U_{x x}=D W(U) \\
U( \pm \infty)=a^{ \pm}
\end{array}\right.
$$

Here $a^{ \pm}$are local minima of a potential $W \in C^{2}\left(\mathbb{R}^{N}\right)$ with $W\left(a^{ \pm}\right)=0$. (1) arises in the theory of phase transitions. Our method is variational but differs from the original artificial constraint method of $[\mathrm{AF}]$ and establishes existence by analysing the loss of compactness in minimising sequences of the action in the appropriate functional space. Our assumptions are slightly different from those considered previously and also imply a priori estimates for the solution.
\end{abstract}

\section{InTRODUCTION.}

In this paper we consider the problem of existence of heteroclinic solutions to the Hamiltonian ODE system

$$
\left\{\begin{array}{l}
U_{x x}=D W(U), \quad U: \mathbb{R} \longrightarrow \mathbb{R}^{N}, \\
U(-\infty)=a^{-}, \quad U(+\infty)=a^{+},
\end{array}\right.
$$

where $W \in C^{2}\left(\mathbb{R}^{N}\right)$ is a potential and $a^{ \pm}$are local minima of it with $W\left(a^{ \pm}\right)=0$. A typical $W$ for $N=2$ is shown in Figures 1,2. Solutions to (1) are known as "heteroclinic connections", being standing waves of the gradient diffusion system

$$
u_{t}=u_{x x}-D W(u), \quad u: \mathbb{R} \times(0,+\infty) \longrightarrow \mathbb{R}^{N} .
$$

(1) arises in the theory of phase transitions. For details we refer to Alikakos-BatesChen $[\mathrm{ABC}]$ and to Alberti [Al]. From the viewpoint of physics, (1) is the Newtonian law of motion with force $-D(-W)$ induced by the potential $-W$ and $U$ the trajectory of a test particle which connects two maxima of $-W$. In the scalar case of $N=1$, existence is textbook material by phase plane methods. For a variational approach we refer to Alberti [Al]. Even in this simple case the unboundedness of $\mathbb{R}$ implies that standard compactness and semicontinuity arguments fail when one tries to obtain solutions to $U_{x x}=W^{\prime}(U)$ variationally as minimisers of the Action functional

$$
E(U)=\int_{\mathbb{R}}\left\{\frac{1}{2}\left|U_{x}\right|^{2}+W(U)\right\} d x .
$$

However, for $N=1$ rearrangement methods do apply (Kawohl [Kaw]). When $N>1,(1)$ is much more difficult. It has first been considered by Sternberg in [St],

2010 Mathematics Subject Classification. Primary 34C37, 46B50, 82B26; Secondary $37 \mathrm{~K} 05$.

Key words and phrases. Heteroclinic connection problem, loss of compactness, phase transitions, Hamiltonian system. 
as a problem arising in the study of the elliptic system $\Delta U=D W(U)$. Noting the compactness problems, he utilises the Jacobi Principle to obtain solutions by studying geodesics in the Riemannian manifold $\left(\mathbb{R}^{N} \backslash\left\{a^{ \pm}\right\}, \sqrt{2 W}\left\langle_{-},{ }_{-}\right\rangle\right)$.

Following a different approach, Alikakos-Fusco [AF] subsequently treated (1) utilising the Least Action Principle. They derived their solutions as minimisers of (3). They introduced an artificial constraint in order to restore compactness and apply the Direct Method and obtained solutions to the (1) by eventually removing the constraint. The same approach has subsequently been applied by Alikakos jointly with the author $[\mathrm{AK}]$ to the respective travelling wave problem for (2), establishing existence of solution to the system $U_{x x}=D W(U)-c U_{x}$ for $c \neq 0$. (1) has attracted some attention in connection with the study of system $\Delta U=D W(U)$ and related material appears also in Alama-Bronsard-Gui [ABG], Bronsard-GuiSchatzman [BGS], Alikakos [A, A2] and Alikakos-Fusco [AF3].

The problem (1) is nontrivial; except for the failure of the Direct Method for (3) due to the loss of compactness, an additional difficulty when $N>1$ is that the Maximum Principle does not apply. In the papers $[\mathrm{AF}],[\mathrm{AK}]$ were introduced substitutes of the Maximum Principle for minimisers. Inspired by these results, the author in [Ka] developed related ideas which apply to general nonconvex functionals. A further difficulty of (1) is that additional minima of $W$ obstruct existence and suitable assumptions on $W$ must be imposed (see [AF]).

In the present work, following [AF], we obtain solutions to (1) as minimisers of (3). We bypass their unilateral constraint method which is of independent interest, but requires a rather delicate analysis. We establish existence for (1) by analysing and then restoring by hand the loss of compactness in minimising sequences. Our motivation comes from the theory of Concentration Compactness (see Lions [L1, L2], and also Bates-Xiaofeng [BX] for a related application of this principle). We note however that Lions' theory merely motivated the ideas utilised herein and we do not know if the well-known condition of "strict inequality" applies in the present context. Our approach is conceptually different: we introduce a functional space tailored for the study of (1) and show that given any minimising sequence of (3), there exist uniformly decaying translates up to which compactness is restored and passage to a minimiser is available (Theorem 2.1). Our main ingredients are certain energy estimates and measure bounds which relate to those of [AF], [AK]. Herein however we utilise a different method: we control the behaviour of the minimising sequence by the sup-level sets $\{W \geq \alpha\}$ and compactify the sequence by suitable translations.

Our basic assumption (A1) is slightly stronger than the respective of [AF], but we still allow for a certain degree of degeneracy. Under this assumption we obtain the a priori quantitative decay estimates $(*)$ by means of energy arguments, without linearising the equation. The rest of the assumptions (A2'), (A2") allow for $W$ 's with several minima and possibly unbounded from below, being similar to those of $[\mathrm{AF}]$. We believe that our proof of the Alikakos-Fusco theorem [AF] provides further insights to the understanding of the problem.

\section{Hypotheses, Setup and the Existence-Compactness Result.}

Hypotheses. We assume $W \in C^{2}\left(\mathbb{R}^{N}\right)$ with $a^{ \pm}$local minima at zero: $W\left(a^{ \pm}\right)=0$. Moreover: 
(A1) There exist $\alpha_{0}, w_{0}>0$ and $\gamma \geq 2$ such that for all $\alpha \in\left(0, \alpha_{0}\right]$ the sublevel sets $\{W \leq \alpha\}$ contain two $C^{2}$ stricitly convex components $\{W \leq \alpha\}^{ \pm}$, each enclosing $a^{ \pm}$respectively such that $\{W=\alpha\}=\partial\{W \leq \alpha\}$ and

$$
W(u) \geq w_{0}\left|u-a^{ \pm}\right|^{\gamma}, \quad u \in\left\{W \leq \alpha_{0}\right\}^{ \pm} .
$$

In addition, at least one of the following two properties is satisfied: either (A2') we have

$$
\left\{W \leq \alpha_{0}\right\}=\left\{W \leq \alpha_{0}\right\}^{+} \bigcup\left\{W \leq \alpha_{0}\right\}^{-},
$$

or

(A2") there exists a convex bounded (localisation) set $\Omega \subseteq \mathbb{R}^{N}$ and a $w_{\max }>\alpha_{0}$ such that $a^{ \pm}$are global minima of $\left.W\right|_{\Omega}$, while

$$
\Omega \subseteq\left\{W \leq w_{\max }\right\}, \quad \partial \Omega \subseteq\left\{W=w_{\max }\right\} .
$$

(A1) allows for $C^{\gamma-\varepsilon}$ flatness at the minima for all $\varepsilon>0$ (but not $C^{\infty}$ flatness as in $[\mathrm{AF}],[\mathrm{AK}])$. The assumption (A2') requires that $\{W \leq \alpha\}^{ \pm}$are the only components of the sublevel sets $\{W \leq \alpha\}$. We note that there is a crucial local monotonicity assumption hidden inside (A1). this monotonicity is included in the statement that the level sets coincide with the boundaries of the sublevel sets and hence "flatness" is exluded.

Under assumption (A2'), we immediately obtain $\liminf _{|u| \rightarrow \infty} W(u) \geq \alpha_{0}$. The assumption (A2") allows for $W$ 's which may be unbounded from below, assuming nonnegativity of $W$ only within $\Omega$.

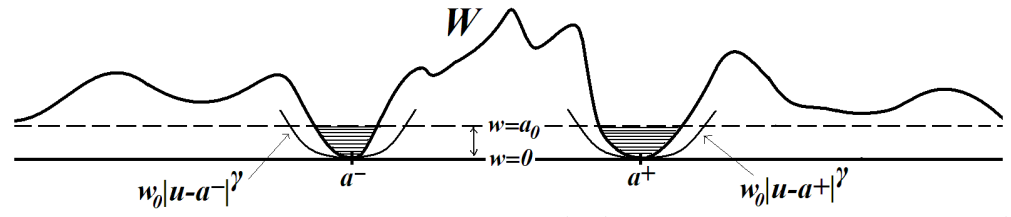

Figure 1: A typical $W$, which satisfies assumption (A1) and the coercivity assumption (A2').

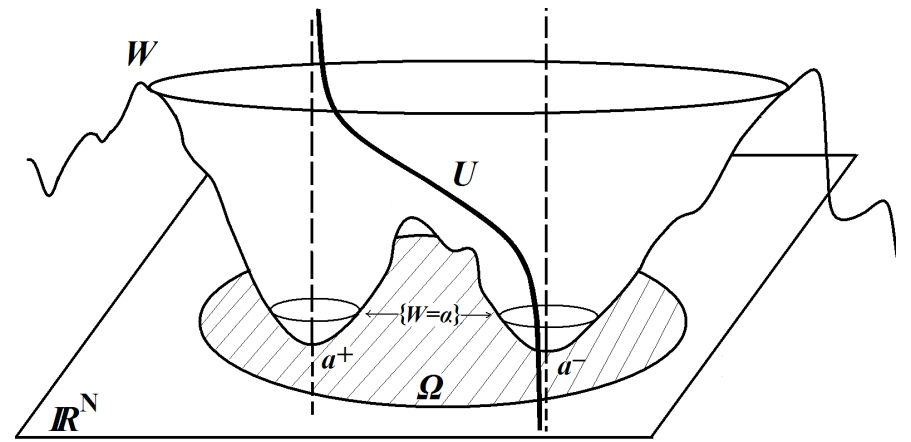

Figure 2: A typical $W$, the heteroclinic solution $U$, the localisation set $\Omega$ of ( $A 2$ "), and the level sets.

Under (A2") the existence of a local minimiser $U$ of (3) with $E(U)>-\infty$ is a certain issue, but (A1) is more crucial. We shall refer to (A2') as the "coercive" and to (A2") as the "non-coercive" assumption.

Functional setup. We derive solutions to (1) as minimisers of (3) in an affine Sobolev space which incorporates the boundary condition $U( \pm \infty)=a^{ \pm}$and excludes the trivial solutions $U=a^{ \pm}$. Let $\left[W_{\mathrm{loc}}^{1, p}(\mathbb{R})\right]^{N}$ denote the local Sobolev space 
of vector functions $U: \mathbb{R} \longrightarrow \mathbb{R}^{N}$. For $\varepsilon>0$ consider the affine function

$$
U_{\mathrm{aff}}^{\varepsilon}(x):= \begin{cases}a^{-}, & x \leq-\varepsilon \\ \left(\frac{\varepsilon-x}{2 \varepsilon}\right) a^{-}+\left(\frac{\varepsilon+x}{2 \varepsilon}\right) a^{-}, & -\varepsilon<x<\varepsilon \\ a^{+}, & x \geq \varepsilon\end{cases}
$$

and set $U_{\text {aff }}^{1}:=U_{\text {aff. }}$ For $p \in(1, \infty)$, the affine $L^{p}$-space, $\left[L_{\text {aff }}^{p}(\mathbb{R})\right]^{N}:=\left[L^{p}(\mathbb{R})\right]^{N}+$ $U_{\text {aff }}$ is a complete metric space for the $L^{p}$ distance. The function (4) will serve also as an a priori upper bound on the action (3) of the minimiser. For $p, q \in(1, \infty)$, we introduce the affine anisotropic Sobolev space

$$
\left[W_{\mathrm{aff}}^{1 ; p, q}(\mathbb{R})\right]^{N}:=\left\{U \in\left[L_{\mathrm{aff}}^{p}(\mathbb{R})\right]^{N}: U_{x} \in\left[L^{q}(\mathbb{R})\right]^{N}\right\} .
$$

(5) is a complete metric space, isometric to a reflexive Banach space. The purpose of this work is to establish the following version of the Alikakos-Fusco theorem from $[\mathrm{AF}]$ :

Theorem 2.1. (Existence - Compactness) Assume that $W$ satisfies (A1) and either (A2') or (A2"), with $\alpha_{0}, \gamma, w_{0}$, as in (A1), (A2'), (A2"). There exists a minimising sequence $\left(U_{i}\right)_{1}^{\infty}$ of the problem

$$
E(U)=\inf \left\{E(V): V \in\left[W_{a f f}^{1 ; \gamma, 2}(\mathbb{R})\right]^{N}\right\}
$$

for (3) with $E\left(U_{i}\right) \geq 0$. For any such $\left(U_{i}\right)_{1}^{\infty}$, there exist $\left(x_{i}\right)_{1}^{\infty} \subseteq \mathbb{R}$ and translates $\widetilde{U}_{i}:=U_{i}\left(\cdot-x_{i}\right)$ which have a subsequence converging weakly in $\left[W_{a f f}^{1 ; \gamma, 2}(\mathbb{R})\right]^{N}$ to a minimiser $U$ which solves (1):

$$
\left\{\begin{array}{l}
U_{x x}=D W(U), \quad U: \mathbb{R} \longrightarrow \mathbb{R}^{N} \\
U(-\infty)=a^{-}, \quad U(+\infty)=a^{+}
\end{array}\right.
$$

In addition, any such minimising solution $U$ satisfies the decay estimates

$$
\left\{\begin{aligned}
\left|U(x)-a^{ \pm}\right| & \leq\left(M w_{0}{ }^{-1}\right)^{\frac{1}{\gamma}}|x|^{-\frac{1}{\gamma}}, & & |x| \geq M \alpha_{0}^{-1} \\
\left|U_{x}(x)\right| & \leq(2 M)^{\frac{1}{2}}|x|^{-\frac{1}{2}}, & & |x| \geq M \alpha_{0}^{-1}
\end{aligned}\right.
$$

as well as the bound $E(U) \leq M$, where

$$
M=\left|a^{+}-a^{-}\right| \max _{\left[a^{-}, a^{+}\right]} \sqrt{2 W} .
$$

Corollary 2.2. $(*)$ imply that the solution is nontrivial. In particular, $U \not \equiv a^{ \pm}$.

Theorem 2.1 asserts that translation invariance of (1) and (3) causes the only possible loss of compactness to minimising sequences. The space $\left[W_{\mathrm{aff}}^{1 ; \gamma, 2}(\mathbb{R})\right]^{N}$ plays a special role to this description. The estimates $(*)$ are an essential property, satisfied uniformly by the compactified sequence of the translates and may not be satisfied by the initial $\left(U_{i}\right)_{1}^{\infty}$. In addition they are quantitative, in the sense that the constant depends explicitly on the potential. Moreover, they guarantee that $U( \pm \infty)=a^{ \pm}$and $U_{x}( \pm \infty)=0$, both fully, not merely up to subsequences. 


\section{Proof of the Main Result.}

Control on the minimising sequence. Let $\left(U_{i}\right)_{1}^{\infty}$ be any minimising sequence of (3). We will tacitly identify each $U_{i}$ with its precise representatives. Since

$$
\left|U\left(x^{\prime \prime}\right)-U\left(x^{\prime}\right)\right| \leq\left(x^{\prime \prime}-x^{\prime}\right)^{\frac{1}{2}}\left(\int_{x^{\prime}}^{x^{\prime \prime}}\left|U_{x}\right|^{2} d x\right)^{\frac{1}{2}},
$$

we have the inclusion $\left[W_{\text {aff }}^{1 ; \gamma, 2}(\mathbb{R})\right]^{N} \subseteq\left[C^{\frac{1}{2}}(\mathbb{R})\right]^{N}$. By (4), we obtain

$$
E\left(U_{\text {aff }}^{\varepsilon}\right)=\int_{-\varepsilon}^{\varepsilon}\left\{\frac{\left|a^{+}-a^{-}\right|^{2}}{8 \varepsilon^{2}}+W\left(\left(\frac{\varepsilon-x}{2 \varepsilon}\right) a^{-}+\left(\frac{\varepsilon+x}{2 \varepsilon}\right) a^{-}\right)\right\} d x
$$

and hence the explicit bounds

$$
\frac{\left|a^{+}-a^{-}\right|^{2}}{4 \varepsilon} \leq E\left(U_{\mathrm{aff}}^{\varepsilon}\right) \leq \frac{\left|a^{+}-a^{-}\right|^{2}}{4 \varepsilon}+2 \varepsilon \max _{\left[a^{-}, a^{+}\right]} W .
$$

We immediately get

$$
\inf _{\left[W_{\text {aff }}^{1 ; \gamma, 2}(\mathbb{R})\right]^{N}} E \leq \inf _{\varepsilon>0} E\left(U_{\text {aff }}^{\varepsilon}\right) \leq\left|a^{+}-a^{-}\right| \max _{\left[a^{-}, a^{+}\right]} \sqrt{2 W}=M<\infty .
$$

$M$ is necessarily a strict upper bound since all $U_{\text {aff }}^{\varepsilon}$ are merely Lipschitz while minimising solutions to (1) must be smooth (this latter fact is a consequence of standard regularity considerations of the solutions to the Euler-Lagrange equations). Further, for $i$ large we have

$$
\int_{\mathbb{R}} \frac{1}{2}\left|\left(U_{i}\right)_{x}\right|^{2} d x+\int_{\mathbb{R}} W\left(U_{i}\right) d x \leq M .
$$

We now derive $\left[L^{\infty}(\mathbb{R})\right]^{N}$ bounds. They are obtained in two different ways, depending on whether (A2') of (A2") is assumed. In the case of (A2'), it is a consequence of the next energy estimate. For $\alpha \in\left(0, \alpha_{0}\right]$ and $i=1,2, \ldots$ we define the control set

$$
\Lambda_{i}^{\alpha}:=\left\{x \in \mathbb{R}: W\left(U_{i}(x)\right)>\alpha\right\} .
$$

Let $|\cdot|$ denote the Lebesgue measure on $\mathbb{R}$ and $M$ the constant in estimates $(*)$.

Lemma 3.1. (Energy Estimate I) Assume $W$ satisfies (A2'). Then we have

$$
\begin{aligned}
M & \geq \alpha\left|\Lambda_{i}^{\alpha}\right|+\frac{1}{2} \int_{\mathbb{R}}\left|\left(U_{i}\right)_{x}\right|^{2} d x, \\
\left\|U_{i}\right\|_{\left[L^{\infty}(\mathbb{R})\right]^{N}} & \leq\left|\Lambda_{i}^{\alpha}\right|^{\frac{1}{2}}\left(\int_{\mathbb{R}}\left|\left(U_{i}\right)_{x}\right|^{2} d x\right)^{\frac{1}{2}}+\max _{u \in\{W \leq \alpha\}^{ \pm}}|u|,
\end{aligned}
$$

for all $i \in \mathbb{N}$.

Proof of Lemma 3.1. By (7) and (8), we have

$$
\begin{aligned}
M \geq E\left(U_{i}\right) & =\int_{\mathbb{R}} W\left(U_{i}\right) d x+\frac{1}{2} \int_{\mathbb{R}}\left|\left(U_{i}\right)_{x}\right|^{2} d x \\
& \geq \int_{\Lambda_{i}^{\alpha}} W\left(U_{i}\right) d x+\frac{1}{2} \int_{\mathbb{R}}\left|\left(U_{i}\right)_{x}\right|^{2} d x \\
& \geq \alpha\left|\Lambda_{i}^{\alpha}\right|+\frac{1}{2} \int_{\mathbb{R}}\left|\left(U_{i}\right)_{x}\right|^{2} d x .
\end{aligned}
$$


This proves (9). Let now $\left(t^{\prime}, t^{\prime \prime}\right)$ be a subinterval of $\Lambda_{i}^{\alpha}$ such that the endpoints $U_{i}\left(t^{\prime}\right), U_{i}\left(t^{\prime \prime}\right)$ of $U_{i}\left(\left(t^{\prime}, t^{\prime \prime}\right)\right)$ lie on different components of $\{W=\alpha\}$. Hence, we have

$$
\left|U_{i}\left(t^{\prime}\right)-U_{i}\left(t^{\prime \prime}\right)\right| \leq\left|t^{\prime \prime}-t^{\prime}\right|^{\frac{1}{2}}\left(\int_{t^{\prime}}^{t^{\prime \prime}}\left|\left(U_{i}\right)_{x}\right|^{2} d x\right)^{\frac{1}{2}} \leq\left|\Lambda_{i}^{\alpha}\right|^{\frac{1}{2}}\left(\int_{\mathbb{R}}\left|\left(U_{i}\right)_{x}\right|^{2} d x\right)^{\frac{1}{2}},
$$

by using that $U_{i}\left(t^{\prime}\right) \in\{W=\alpha\}^{ \pm}$, we deduce

$$
\left|U_{i}\left(t^{\prime \prime}\right)-U_{i}\left(t^{\prime}\right)\right| \geq\left|U_{i}\left(t^{\prime \prime}\right)\right|-\left|U_{i}\left(t^{\prime}\right)\right| \geq\left|U_{i}\left(t^{\prime \prime}\right)\right|-\max _{u \in\{W \leq \alpha\}^{ \pm}}|u| .
$$

This establishes estimate (10), proving Lemma 3.1.

Corollary 3.2. ( $L^{\infty}$ bound under (A2')) If $W$ satisfies (A1), (A2'), then

$$
\sup _{i \geq 1}\left\|U_{i}\right\|_{\left[L^{\infty}(\mathbb{R})\right]^{N}} \leq \sqrt{\frac{2}{\alpha_{0}}} M+\max _{u \in\left\{W \leq \alpha_{0}\right\}^{ \pm}}|u| .
$$

Now we turn to the case of (A2"). We obtain existence of a minimising sequence $\left(U_{i}\right)_{1}^{\infty}$ of (3) localised inside $\bar{\Omega} \subseteq \mathbb{R}^{N}$ whereon $\left.W\right|_{\Omega} \geq 0$.

Lemma 3.3. ( $L^{\infty}$ bound under (A2")) If $W$ satisfies (A1), (A2"), there is a minimising sequence $\left(U_{i}\right)_{1}^{\infty}$ for which $\bigcup_{i=1}^{\infty} U_{i}(\mathbb{R}) \subseteq \bar{\Omega}$ and $W\left(U_{i}\right) \geq 0$. Moreover,

$$
\sup _{i \geq 1}\left\|U_{i}\right\|_{\left[L^{\infty}(\mathbb{R})\right]^{N}} \leq \max _{u \in \partial \Omega}|u| .
$$

Proof of Lemma 3.3. We show the existence of a deformation of $W$ to a new $\bar{W}$ such that $\bar{W}=W$ on $\Omega$ and all the minimising sequences of (3) relative to $\bar{W}$ in $\left[W_{\mathrm{aff}}^{1 ; \gamma, 2}(\mathbb{R})\right]^{N}$ can be chosen to be localised inside $\Omega$. By (A2"), $W \leq w_{\max }$ inside $\Omega$ and $W=w_{\max }$ on $\partial \Omega$. We define $\bar{W}$ by reflecting with respect to the hyperplane $\left\{w=w_{\max }\right\}$ the portions of the graph of $W$ which lie in the halfspace $\left\{w<w_{\max }\right\}$, to $\left\{w>w_{\max }\right\}$.

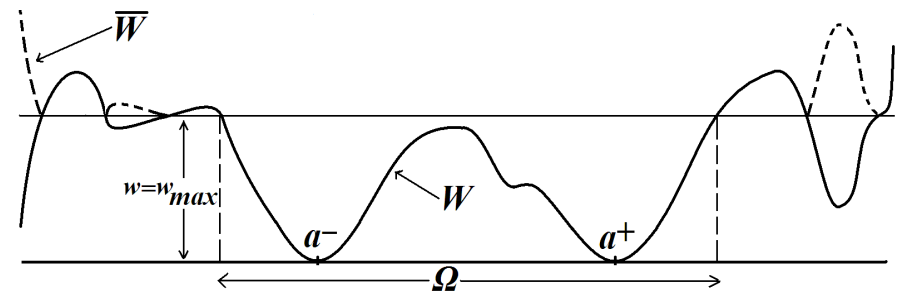

Figure 3: The deformed coercive potential $\bar{W}$, for which $w=w_{\max }$ is a lower bound outside of $\Omega$.

By construction, $\bar{W}(u) \geq w_{\max }$, for $u \in \mathbb{R}^{N} \backslash \Omega$. Suppose for the shake of contradiction that $\bar{W}$ has a minimising sequence $\left(U_{i}\right)_{1}^{\infty}$ such that for some $U_{i}$ and $a<b$, $U_{i}((a, b)) \subseteq \mathbb{R}^{N} \backslash \Omega$. This is the only case that has to be excluded since by the definition of $\left[W_{\text {aff }}^{1 ; \gamma, 2}(\mathbb{R})\right]^{N}$ the "tails" of each $U_{i}$ approach asymptotically $a^{ \pm} \in \Omega$, at least along a sequence (in general of course there may exists countably many such intervals and we apply this argument to each one of them). By replacing $U_{i}([a, b])$ by the straight line segment with the same endpoints, i.e. by defining

$$
\bar{U}_{i}(x):= \begin{cases}U_{i}(x), & x \in \mathbb{R} \backslash(a, b), \\ \left(\frac{x-a}{b-a}\right) U_{i}(b)+\left(\frac{b-x}{b-a}\right) U_{i}(a), & x \in(a, b),\end{cases}
$$


we obtain by convexity of $\Omega$ that $\bar{U}_{i}(\mathbb{R}) \subseteq \bar{\Omega}$. By pointwise comparison,

$$
\int_{a}^{b} \bar{W}\left(\bar{U}_{i}(x)\right) d x \leq \int_{a}^{b} \bar{W}\left(U_{i}(x)\right) d x .
$$

In addition, $\left.\bar{U}_{i}\right|_{(a, b)}$ minimises the Dirichlet integral since it is a straight line, thus

$$
\frac{\left|\bar{U}_{i}(b)-\bar{U}_{i}(a)\right|^{2}}{b-a}=\int_{a}^{b}\left|\left(\bar{U}_{i}\right)_{x}\right|^{2} d x<\int_{a}^{b}\left|\left(U_{i}\right)_{x}\right|^{2} d x .
$$

(14) and (15) imply that there exists a minimising sequence of the Action (3) with the potential $\bar{W}$ in the place of $W$ which lies inside $\bar{\Omega}$. Finally, $\left.W\right|_{\Omega}=\left.\bar{W}\right|_{\Omega}$ by construction.

In the case that (A2") is assumed, we fix a sequence valued inside $\Omega$. Moreover,

$$
M \geq \liminf _{i \rightarrow \infty} E\left(U_{i}\right)=: \inf \left\{E(V): V \in\left[W_{\mathrm{aff}}^{1 ; \gamma, 2}(\mathbb{R})\right]^{N}\right\} \geq 0 .
$$

As the notation suggests, the right hand side will henceforth stand for $\liminf _{i \rightarrow \infty}\left(U_{i}\right)$. Now we employ (A1) to show that $\Lambda_{i}^{\alpha}$ is connected. For $\alpha \in\left(0, \alpha_{0}\right], i=1,2, \ldots$, we set

$$
\lambda_{i}^{\alpha-}:=\inf \Lambda_{i}^{\alpha}, \quad \lambda_{i}^{\alpha+}:=\sup \Lambda_{i}^{\alpha}
$$

We also set

$$
d_{\alpha}:=\operatorname{dist}\left(\{W=\alpha\}^{-},\{W=\alpha\}^{+}\right)
$$

We note that $d_{\alpha}$ is the distance between the 2 components of the level set $\{W=\alpha\}$.

Lemma 3.4. (Control on the $\lambda^{\alpha \pm}$ times) Assume $W$ satisfies (A1) and either (A2') or (A2"). Then, for $\alpha \in\left(0, \alpha_{0}\right]$, if $\left(U_{i}\right)_{1}^{\infty}$ is the minimising sequence constructed previously, then the respective sets $\Lambda_{i}^{\alpha}$ are intervals and hence

$$
\Lambda_{i}^{\alpha}=\left(\lambda_{i}^{\alpha-}, \lambda_{i}^{\alpha+}\right) .
$$

Proof of Lemma 3.4. The claim follows by a direct application of the Replacement Lemma 12 in p. 1381 of [AK] by choosing as $\mu$ the Lebesgue measure on $\mathbb{R}$. In order to make the presentation self-contained, we provide also an alternative proof which bypasses this maximum principle type of result of $[A K]$. We note that the result follows by the replacement lemma of $[\mathrm{AF}]$ as well, but this is not entirely direct since herein we use convex level sets and not balls.

We fix a term $U_{i}$ of the minimising sequence and a respective $\Lambda_{i}^{\alpha}$ and we drop the subscript $i$. Since $\Lambda^{\alpha}=\{W(U)>\alpha\}$ is open, there exist countably many open intervals such that

$$
\Lambda^{\alpha}=\bigcup_{p=0}^{\infty}\left(x_{2 p}^{\alpha}, x_{2 p+1}^{\alpha}\right) .
$$

Since $U \in\left[C^{0}(\mathbb{R})\right]^{N}$, each image $U\left(\left(x_{2 p}^{\alpha}, x_{2 p+1}^{\alpha}\right)\right)$ is connected with endpoints on $\{W(U)=\alpha\}$ and

$$
U\left(\Lambda^{\alpha}\right)=\bigcup_{p=0}^{\infty} U\left(\left(x_{2 p}^{\alpha}, x_{2 p+1}^{\alpha}\right)\right)
$$


Claim 3.5. For all $p \in \mathbb{N}$, the image $U\left(\left(x_{2 p}^{\alpha}, x_{2 p+1}^{\alpha}\right)\right)$ has endpoints on different components $\{W(U)=\alpha\}^{ \pm}$of $\{W(U)=\alpha\}$.

Indeed, supose for the sake of contradiction that for some $p$, both $U\left(x_{2 p}^{\alpha}\right)$ and $U\left(x_{2 p+1}^{\alpha}\right)$ are on $\{W(U)=\alpha\}^{+}$. The deformation of Lemma 3.3 together with the strictness of assumption (A1) contradicts minimality of $U$. The same holds if the endpoints are on $\{W(U)=\alpha\}^{-}$. The claim follows.

Claim 3.6. The set $\Lambda^{\alpha}$ consists of finitely many intervals of odd number.

By Claim 3.5, for each $p, U\left(\left(x_{2 p}^{\alpha}, x_{2 p+1}^{\alpha}\right)\right)$ has endpoints on different components $\{W(U)=\alpha\}$. Hence, in view (17) we have

$$
d_{\alpha} \leq\left|U\left(x_{2 p+1}^{\alpha}\right)-U\left(x_{2 p}^{\alpha}\right)\right| \leq \int_{x_{2 p}^{\alpha}}^{x_{2 p+1}^{\alpha}}\left|U_{x}\right|
$$

and hence for each $q \in \mathbb{N}$, by (18),

$$
q d_{\alpha} \leq \sum_{p=0}^{q} \int_{x_{2 p}^{\alpha}}^{x_{2 p+1}^{\alpha}}\left|U_{x}\right| \leq \int_{\Lambda^{\alpha}}\left|U_{x}\right| \leq\left|\Lambda^{\alpha}\right|^{1 / 2}\left(\int_{\mathbb{R}}\left|U_{x}\right|^{2}\right)^{1 / 2} .
$$

Hence, by Lemma 3.1, we have

$$
q \leq \frac{1}{d_{\alpha}}\left(\frac{M}{\alpha}\right)^{1 / 2} M^{1 / 2}
$$

which implies that there exists a $p_{\alpha} \in \mathbb{N}$ no greater than the integer part of $M / \sqrt{\alpha} d_{\alpha}$ such that

$$
\Lambda^{\alpha}=\bigcup_{p=0}^{p_{\alpha}}\left(x_{2 p}^{\alpha}, x_{2 p+1}^{\alpha}\right)
$$

Since

$$
\mathbb{R} \backslash \Lambda^{\alpha}=\left(-\infty, x_{0}^{\alpha}\right] \bigcup\left[x_{1}^{\alpha}, x_{2}^{\alpha}\right] \bigcup \ldots \ldots \bigcup\left[x_{2 p^{\alpha}-1}^{\alpha}, x_{2 p^{\alpha}}^{\alpha}\right] \bigcup\left[x_{2 p_{\alpha}+1}^{\alpha},+\infty\right)
$$

and $\mathbb{R} \backslash \Lambda^{\alpha}$ equals $\{W(U) \leq \alpha\}, U$ exits $\{W(U) \leq \alpha\}^{-}$for the 1st time at $x=x_{0}^{\alpha}$ and stays inside $\{W(U) \leq \alpha\}^{+}$after $x=x_{2 p_{\alpha}+1}^{\alpha}$ (Figure 4). Since

$$
\begin{aligned}
U\left(x_{0}^{\alpha}\right) & \in\{W=\alpha\}^{-}, \\
U\left(x_{1}^{\alpha}\right), U\left(x_{2}^{\alpha}\right) & \in\{W=\alpha\}^{+}, \\
U\left(x_{3}^{\alpha}\right), U\left(x_{4}^{\alpha}\right) & \in\{W=\alpha\}^{-},
\end{aligned}
$$

in view of (19) the number of interval has to odd, for otherwise $U$ stays inside $\{W \leq \alpha\}^{-}$for infinite time and this contradicts that (at least along a sequence) $U(x)$ converges to $a^{+}$as $x \rightarrow \infty$.

Claim 3.7. All subsets $U\left(\left(x_{1}^{\alpha}, x_{2}^{\alpha}\right)\right), U\left(\left(x_{3}^{\alpha}, x_{4}^{\alpha}\right)\right), \ldots, U\left(\left(x_{2 p_{\alpha}-1}^{\alpha}, x_{2 p_{\alpha}}^{\alpha}\right)\right)$ of the image $U\left(\mathbb{R} \backslash \Lambda^{\alpha}\right)$ lie inside the interior $\{W<\alpha\}$ and can not touch the boundary $\{W=\alpha\}$ (Figure 4). 
Fix a $q \in\left\{1, \ldots, p_{\alpha}\right\}$ and assume for the sake of contradiction that there is $[a, b] \subseteq$ $\left(x_{2 q-1}^{\alpha}, x_{2 q}^{\alpha}\right)$, such that $U([a, b])$ lies on the boundary $\{W=\alpha\}$. Then, by replacing $U([a, b])$ by the straight line segment with the same endpoints (as in Lemma 3.1), we obtain a contradiction.

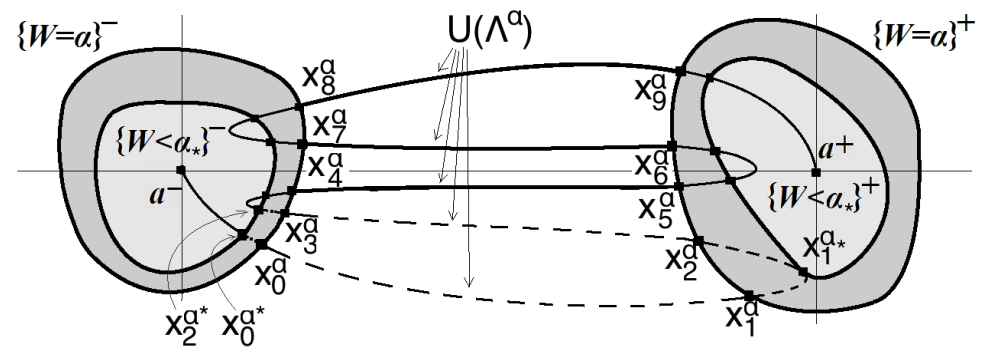

Figure 4: Illustration with $p_{\alpha}=4$. By minimality the dashed line with endpoints $U\left(x_{0}^{\alpha *}\right), U\left(x_{2}^{\alpha *}\right)$ can not exist. For brevity we have denoted the points $U\left(x_{p}^{\alpha}\right)$ by $x_{p}^{\alpha}$.

Hence, if $U\left(\left(x_{2 q-1}^{\alpha}, x_{2 q}^{\alpha}\right)\right)$ touches the boundary $\{W=\alpha\}$, this happens at isolated points (and otherwise it is inside $\{W<\alpha\}$ ).

Fix such a point and call it $x^{*}$. By continuity and by assumption (A1), there exist $\delta^{0}, \delta^{ \pm}>0$ such that $U\left(\left(x^{*}-\delta^{-}, x^{*}+\delta^{+}\right)\right)$lies outside $\left\{W<\alpha-\delta^{0}\right\}$.

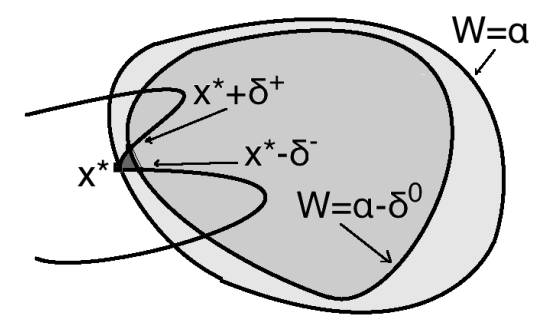

Figure 5.

By replacing $U\left(\left(x^{*}-\delta^{-}, x^{*}+\delta^{+}\right)\right)$by the straight line segment with the same endpoints (as in Lemma 3.1), we obtain a contradiction. By arguing for all such points $x^{*}$, we see that $U\left(\left(x_{2 q-1}^{\alpha}, x_{2 q}^{\alpha}\right)\right)$ lies inside $\{W<\alpha\}$, as desired.

Claim 3.8. $p_{\alpha}=0$, that is $\Lambda^{\alpha}$ has only one connected component and hence $x_{1}^{\alpha}=x_{2 p_{\alpha}+1}^{\alpha}$.

We argue by contradiction. Suppose that $p \in\left\{1, \ldots, p_{\alpha}\right\}$ and consider the set

$$
A:=\left\{\beta \in(0, \alpha) \mid U\left(\left(x_{2 p-1}^{\alpha}, x_{2 p}^{\alpha}\right)\right) \bigcap_{p=1}^{p_{\alpha}}\{W<\beta\} \neq \emptyset\right\} .
$$

Since $U\left(\left(x_{2 p-1}^{\alpha}, x_{2 p}^{\alpha}\right)\right.$ lies strictly inside the sublevel set, we have that $A \neq \emptyset$. We set

$$
\alpha_{*}:=\inf A .
$$

Since the components $U\left(\left(x_{2 p-1}^{\alpha}, x_{2 p}^{\alpha}\right)\right)$ are finitely many, their distance from the minimum of $W$ is bounded away frow zero and hence $0<\alpha_{*}<\alpha$. By definition of $\alpha_{*}$, there exists at least one of the components $U\left(\left(x_{2 p-1}^{\alpha}, x_{2 p}^{\alpha}\right)\right)$, say for $p=1$, which touches only the boundary of $\left\{W=\alpha_{*}\right\}=\partial\left\{W<\alpha_{*}\right\}$ and does not intersect 
$\left\{W<\alpha_{*}\right\}$. Morover, it can not touch the boundary at more than one points. Hence,

$$
\Lambda^{\alpha_{*}}=\left(x_{0}^{\alpha_{*}}, x_{1}^{\alpha_{*}}\right) \bigcup\left(x_{0}^{\alpha_{*}}, x_{1}^{\alpha_{*}}\right) \bigcup \ldots
$$

and consequently $U\left(\left(x_{0}^{\alpha_{*}}, x_{2}^{\alpha_{*}}\right)\right)$ is contained into $\left\{W \geq \alpha_{*}\right\}$ and only $U\left(x_{1}^{\alpha_{*}}\right)$ is on $\left\{W=\alpha_{*}\right\}^{+}$, having both the endpoints $U\left(x_{0}^{\alpha_{*}}\right), U\left(x_{2}^{\alpha_{*}}\right)$ on $\left\{W=\alpha_{*}\right\}^{-}$. By arguing as in Lemma 3.1 for $\left.U\right|_{\left(x_{0}^{\alpha_{*}}, x_{2}^{\alpha_{*}}\right)}$, we obtain a contradiction to the minimality of the action of $U$. Hence, $p_{\alpha}=0$.

By putting Claims 3.5, 3.6, 3.7 and 3.8, we see that Lemma 3.4 has been established.

The following sharpens (9), under the additional information that $\Lambda_{i}^{\alpha}$ is connected.

Lemma 3.9. (Energy estimate II) For all $\alpha \in\left(0, \alpha_{0}\right]$ and $i \geq 1$, we have

$$
M \geq E\left(U_{i}\right) \geq \frac{d_{\alpha}{ }^{2}}{2\left(\lambda_{i}^{\alpha+}-\lambda_{i}^{\alpha-}\right)}+\alpha\left(\lambda_{i}^{\alpha+}-\lambda_{i}^{\alpha-}\right) .
$$

Proof of Lemma 3.9. Proceeding as in Lemma 3.1, we recall (7) to obtain

$$
M \geq E\left(U_{i}\right) \geq \alpha\left(\lambda_{i}^{\alpha+}-\lambda_{i}^{\alpha-}\right)+\frac{1}{2} \int_{\lambda_{i}^{\alpha-}}^{\lambda_{i}^{\alpha+}}\left|\left(U_{i}\right)_{x}\right|^{2} d x
$$

where we have also used Lemma 3.4. In addition,

$$
d_{\alpha} \leq\left|U_{i}\left(\lambda_{i}^{\alpha-}\right)-U_{i}\left(\lambda_{i}^{\alpha+}\right)\right| \leq\left(\lambda_{i}^{\alpha+}-\lambda_{i}^{\alpha-}\right)^{\frac{1}{2}}\left(\int_{\lambda_{i}^{\alpha}}^{\lambda_{i}^{\alpha+}}\left|\left(U_{i}\right)_{x}\right|^{2} d x\right)^{\frac{1}{2}} .
$$

The Lemma follows.

Corollary 3.10. (Uniform bounds on $\left|\Lambda_{i}^{\alpha}\right|$ ) For $i=1,2, \ldots, \alpha \in\left[0, \alpha_{0}\right]$, we have

$$
\frac{d_{\alpha}^{2}}{2 M} \leq\left|\Lambda_{i}^{\alpha}\right|=\lambda_{i}^{\alpha+}-\lambda_{i}^{\alpha-} \leq \frac{M}{\alpha}
$$

Restoration of Compactness. The bounds (22) provide information which allow to control the behaviour of each $U_{i}$ by "tracking" the $\Lambda_{i}^{\alpha}$ 's. In the terminology of $[\mathrm{ABG}]$, translation invariance of $(3)$ and (1) allows us to "fix a centre" for the $U_{i}$ 's and align the minimising sequence, preventing the terms from escaping to $\pm \infty$. For $i=1,2, \ldots$, we set

$$
x_{i}:=\frac{\lambda_{i}^{\alpha_{0}+}+\lambda_{i}^{\alpha_{0}-}}{2}
$$

which is the centre of the control set $\Lambda_{i}^{\alpha_{0}}=\left(\lambda_{i}^{\alpha_{0}-}, \lambda_{i}^{\alpha_{0}+}\right)$. We define the translates of the minimising sequence $\left(U_{i}\right)_{1}^{\infty}$ by:

$$
\widetilde{U}_{i}:=U_{i}\left(\cdot-x_{i}\right), \quad i=1,2, \ldots
$$

For these translates, their control sets $\widetilde{\Lambda}_{i}^{\alpha_{0}}=\left(\widetilde{\lambda}_{i}^{\alpha_{0}-}, \widetilde{\lambda}_{i}^{\alpha_{0}+}\right)$ are centred at $x=0$, being symmetric (Figure 5). The control sets $\widetilde{\Lambda}_{i}^{\alpha}$ of $\widetilde{U}_{i}$ and $\Lambda_{i}^{\alpha}$ of $U_{i}$ are related by

$$
\left(\widetilde{\lambda}_{i}^{\alpha-}, \widetilde{\lambda}_{i}^{\alpha+}\right)=\widetilde{\Lambda}_{i}^{\alpha}=\left(\lambda_{i}^{\alpha-}-x_{i}, \lambda_{i}^{\alpha+}-x_{i}\right)
$$


The translates $\left(\widetilde{U}_{i}\right)_{1}^{\infty}$ defined by $(23),(24)$ will be referred to as the compactified sequence relative to the initial $\left(U_{i}\right)_{1}^{\infty}$. The sequence $\left(\widetilde{U}_{i}\right)_{1}^{\infty}$ will turn out to be weakly precompact in $\left[W_{\text {aff }}^{1 ; \gamma, 2}(\mathbb{R})\right]^{N}$, converging to a solution of (1).

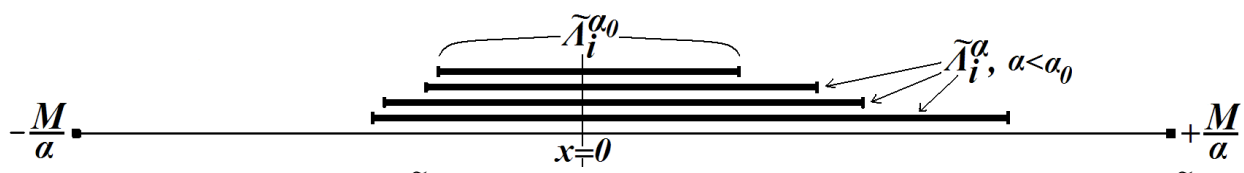

Figure 5: The control sets of $\widetilde{U}_{i}$ are symmetric for $\alpha=\alpha_{0}$. For $\alpha<\alpha_{0}$ may not be, but $0 \in \widetilde{\Lambda}_{i}^{\alpha}$.

Corollary 3.11. (Uniform bounds for the compactified sequence) For $i=$ $1,2, \ldots$ and $\alpha \in\left(0, \alpha_{0}\right],(22)$ can be rewritten in view of $(23),(24),(25)$ as

$$
\frac{d_{\alpha}^{2}}{2 M} \leq\left|\widetilde{\Lambda}_{i}^{\alpha}\right|=\widetilde{\lambda}_{i}^{\alpha+}-\widetilde{\lambda}_{i}^{\alpha-} \leq \frac{M}{\alpha} .
$$

In particular, since $0 \in \widetilde{\Lambda}_{i}^{\alpha}$ for $\alpha \in\left(0, \alpha_{0}\right]$ and $i=1,2, \ldots$, we have

$$
\max \left\{\left|\tilde{\lambda}_{i}^{\alpha+}\right|,\left|\tilde{\lambda}_{i}^{\alpha-}\right|\right\} \leq \frac{M}{\alpha}
$$

Bounds and Decay Estimates for the Compactified Sequence. The $\left[L^{2}(\mathbb{R})\right]^{N}$ bound on the derivatives $\left(\widetilde{U}_{i}\right)_{x}$ is immediate by the kinetic energy term of $(3)$. The more interesting uniform $\left[L_{\text {aff }}^{\gamma}(\mathbb{R})\right]^{N}$ bound is a consequence of our assumption (A1) on the nonconvex potential term.

Lemma 3.12. (Estimates for the compactified sequence) Let $\left(\widetilde{U}_{i}\right)_{1}^{\infty}$ be given by (23) and (24). If $W$ satisfies (A1) and either (A2') or (A2"), then $\left(\widetilde{U}_{i}\right)_{1}^{\infty}$ lies in a ball of $\left[W_{\text {aff }}^{1 ; \gamma, 2}(\mathbb{R})\right]^{N} \cap\left[L^{\infty}(\mathbb{R})\right]^{N}$ centred at $U_{\text {aff. }}$ Moreover,

$$
\begin{gathered}
\sup _{i \geq 1}\left\|\widetilde{U}_{i}-U_{a f f}\right\|_{\left[L^{\gamma}(\mathbb{R})\right]^{N}} \leq M^{\frac{1}{\gamma}}\left\{\frac{1}{w_{0}}+\frac{2}{\alpha_{0}}\left\{\sup _{i \geq 1}\left\|\widetilde{U}_{i}\right\|_{\left[L^{\infty}(\mathbb{R})\right]^{N}}\right\}^{\gamma}\right\}^{\frac{1}{\gamma}} \\
\sup _{i \geq 1}\left\|\widetilde{U}_{i}\right\|_{\left[L^{\infty}(\mathbb{R})\right]^{N}} \leq \begin{cases}\sqrt{\frac{2}{\alpha_{0}}} M+\max _{u \in\left\{W \leq \alpha_{0}\right\}^{ \pm}}|u|, \quad \text { under }\left(A 2^{\prime}\right) \\
\max _{u \in \partial \Omega}|u|, & \text { under }\left(A 2^{\prime \prime}\right)\end{cases} \\
\sup _{i \geq 1}\left\|\left(\widetilde{U}_{i}\right)_{x}\right\|_{\left[L^{2}(\mathbb{R})\right]^{N}} \leq \sqrt{2 M .}
\end{gathered}
$$

Proof of Lemma 3.12. (30) follows from translation invariance, while (29) follows by (11), (12) and translation invariance. Thus, we only need to prove (28). For,

$$
M \geq \int_{\mathbb{R}} W\left(U_{i}\right) d x=\int_{\mathbb{R}} W\left(\widetilde{U}_{i}\right) d x \geq \int_{-\infty}^{-\frac{M}{\alpha}} W\left(\widetilde{U}_{i}\right) d x+\int_{+\frac{M}{\alpha}}^{+\infty} W\left(\widetilde{U}_{i}\right) d x .
$$

Utilizing (27), we obtain $W\left(\widetilde{U}_{i}(x)\right) \leq \alpha$, for $i=1,2, \ldots$ when $|x| \geq M \alpha^{-1}$. Thus, for such $x$ we are in the domain of validity of (A1). For $\alpha=\alpha_{0}$, we get

$$
w_{0}\left(\int_{-\infty}^{-\frac{M}{\alpha_{0}}}\left|\widetilde{U}_{i}-a^{-}\right|^{\gamma} d x+\int_{+\frac{M}{\alpha_{0}}}^{+\infty}\left|\widetilde{U}_{i}-a^{+}\right|^{\gamma} d x\right) \leq M
$$


By restricting to smaller $\alpha \leq \alpha_{1}\left(<\alpha_{0}\right)$, we may assume that $\left(-M \alpha_{0}{ }^{-1},+M \alpha_{0}{ }^{-1}\right) \supseteq$ $(-1,1)$. Hence, $U_{\text {aff }}=a^{ \pm}$for $|x| \geq M \alpha_{0}^{-1}$. To conclude, we employ (29) to get

$$
\int_{-\frac{M}{\alpha_{0}}}^{+\frac{M}{\alpha_{0}}}\left|\widetilde{U}_{i}-U_{\mathrm{aff}}\right|^{\gamma} d x \leq \frac{2 M}{\alpha_{0}}\left\{\left\|\widetilde{U}_{i}\right\|_{\left[L^{\infty}(\mathbb{R})\right]^{N}}\right\}^{\gamma} .
$$

Putting these estimates together, we see that (28) has been established.

Lemma 3.13. (Uniform decay estimate) If $W$ satisfies (A1), the compactified sequence $\left(\widetilde{U}_{i}\right)_{1}^{\infty}$ satisfies $\left|\widetilde{U}_{i}(x)-a^{ \pm}\right| \leq\left(M w_{0}{ }^{-1}\right)^{\frac{1}{\gamma}}|x|^{-\frac{1}{\gamma}}$, for $|x| \geq M \alpha_{0}{ }^{-1}$.

Proof of Lemma 3.13. We have already seen in Lemma 3.12 that (27) implies $W\left(\widetilde{U}_{i}(x)\right) \leq \alpha$, for $i=1,2, \ldots$ when $|x| \geq M \alpha^{-1}$. By (A1), we have

$$
w_{0}\left|\widetilde{U}_{i}(x)-a^{ \pm}\right|^{\gamma} \leq W\left(\widetilde{U}_{i}(x)\right),
$$

for all such $x \in \mathbb{R}$. Therefore,

$$
\left|\widetilde{U}_{i}(x)-a^{ \pm}\right|^{\gamma} \leq \frac{\alpha}{w_{0}}
$$

for all $|x| \geq M \alpha^{-1}$ and all $\alpha \leq \alpha_{0}$. We fix an $x \in \mathbb{R}$ for which $|x| \geq M \alpha_{0}^{-1}$ and choose

$$
\alpha(x):=\frac{|x|}{M} .
$$

This is a legitimate choice since $|x|=M \alpha(x)^{-1} \geq M \alpha_{0}{ }^{-1}$. We thus obtain that

$$
\left|\widetilde{U}_{i}(x)-a^{ \pm}\right|^{\gamma} \leq \frac{\alpha(x)}{w_{0}} \leq \frac{M}{w_{0}|x|}
$$

and by letting $x$ vary, the estimate follows.

Corollary 3.14. (A priori decay estimates) Assume $W$ satisfies (A1). Then, if a solution $U$ to (1) exists, it must satisfy estimates $(*)$ of Theorem 2.1.

Proof of Corollary 3.14. We recall from [AF] the equipartition property $\left|U_{x}\right|^{2}=$ $2 W(U)$ satisfied by solutions of (1). Equipartition implies $\left|U_{x}\right|^{2}=2 W(U) \leq 2 \alpha$, for $|x| \geq M \alpha^{-1}$ and $\alpha \leq \alpha_{0}$. The rest follows closely the proof of Lemma 3.13.

Passage to a minimising solution. We conclude by proving existence of minimisers. By (28), (29) and (30), the sequence of translates $\left(\widetilde{U}_{i}\right)_{1}^{\infty}$ converges to some $U$ weakly in $\left[W_{\text {aff }}^{1 ; \gamma, 2}(\mathbb{R})\right]^{N}$ along a subsequence. By denoting the subsequence again by $\left(\widetilde{U}_{i}\right)_{1}^{\infty}$, we have that $\widetilde{U}_{i}-U \longrightarrow 0$ in $\left[L^{\gamma}(\mathbb{R})\right]^{N}$ and $\left(\widetilde{U}_{i}-U\right)_{x} \longrightarrow 0$ in $\left[L^{2}(\mathbb{R})\right]^{N}$, both as $i \rightarrow \infty$. Up to a further subsequence, we have $\widetilde{U}_{i} \longrightarrow U$ in $\left[L_{\text {loc }}^{2}(\mathbb{R})\right]^{N}$ and a.e. on $\mathbb{R}$ as $i \rightarrow \infty$. By the weak lower semicontinuity of the $L^{2}$ norm and the Fatou Lemma, we obtain

$$
E(U) \leq \liminf _{i \rightarrow \infty} E\left(\widetilde{U}_{i}\right)
$$

By (6), we also get $0 \leq E(U) \leq M$. Thus $U$ is a local minimiser of the functional $(3)$ in $\left[W_{\text {aff }}^{1 ; \gamma, 2}(\mathbb{R})\right]^{N}$. Hence, $U$ solves $(1)$ classically and satisfies the estimates $(*)$. The proof of Theorem 2.1 is complete.

Acknowledgement. We are indebted to N. Alikakos for the careful reading of an earlier version of this manuscript and for providing his valuable suggestions which 
led to substantial improvements of the content of the paper. We would also like to thank the referee of the paper for their comments and suggestions.

\section{REFERENCES}

[ABG] S. Alama, L. Bronsard, C. Gui, Stationary layered solutions in $\mathbb{R}^{2}$ for an Allen - Cahn system with multiple well potential, Cal. Var. 5, (1997), 359 - 390.

[Al] Alberti G. Variational models for phase transitions, an approach via $\Gamma$-convergence, in L. Ambrosio and N. Dancer, Calculus of Variations and Partial Differential Equations. Springer Verlag, Berlin, 2000, 95 - 114.

[A] N. Alikakos, A new proof for the existence of an equivariant entire solution connecting the minima of the potential for the system $u \quad W u(u)=0$, Comm. Partial Diff. Eqs 37 No. 12 (2012) pp. 2093 - 2115.

[A2] N. Alikakos, Some basic facts on the system $u W_{u}(u)=0$ Proc. Amer. Math. Soc. 139 (2011) pp. 153 - 162.

$[\mathrm{ABC}]$ N. Alikakos, P. W. Bates, X. Chen, Convergence of the Cahn-Hilliard equation to the Hele-Shaw model, Archive for Rational Mechanics and Analysis 20. XII. 1994, Volume 128, Issue 2, pp 165-205.

[AF] N. Alikakos, G. Fusco, On the connection problem for potentials with several global minima, Ind. J. of Math, Vol. 57, No. 4, 1871 - 1906, (2008).

[AF3] N. Alikakos, G. Fusco, Entire solutions to equivariant elliptic systems with variational structure, Arch. Rat. Mech. Anal. 202 No. 2 (2011) pp. 567 - 597.

[AK] N. Alikakos, N. Katzourakis, Heteroclinic Travelling Waves of Gradient Diffusion Systems, Transactions of the AMS, Vol. 363, Nr 3 (2011), Pages 1365 - 1397.

[BX] P. W. Bates, R. Xiaofeng, Transition layer solutions of a higher order equation in an infinite tube, Communications in PDE, 21:1, 109 - 145, 1996.

[BGS] L. Bronsard, C. Gui, M. Schatzman A Three-Layered minimiser in $\mathbb{R}^{2}$ for a Variational Problem with a Symmetric Three-Well Potential, Com. P. Appl. Math. Vol. XLIX, (1996), $687-715$.

[Ka] N. Katzourakis, Maximum Principles for Vectorial Approximate Minimizers of Nonconvex Functionals, Calculus of Variations and PDE, DOI 10.1007/s00526-012-0491-6, published online 01 Feb. 2012.

[Kaw] B. Kawohl, Rearrangement and Convexity of Level Sets in PDE. Lectures Notes in Mathematics 1150. Springer-Verlag, Berlin-Heidelberg, 1985.

[L1] P. L. Lions, The concentration compactness principle in the calculus of variations. The locally compact case, part 1, Ann. Inst. H. Poincaré C, Tom 1, No 2, p. 109 - 145, 1984.

[L2] P. L. Lions, The concentration compactness principle in the calculus of variations. The limit case, part 1, Revista Matemátika Iberoamericana, Vol. 1, No 1, 145 - 201, 1985.

[St] P. Sternberg, Vector valued local minimisers of nonconvex variational problems, Rocky Mountain J. of Math., 21, (1991), no. 2, 799 - 807.

Department of Mathematics and Statistics, University of Reading, Whiteknights CAmpus, PO Box 220, RG6 6AX, REAding, UK

E-mail address: n.katzourakis@reading.ac.uk 